\title{
Effectiveness of a bed alarm system to predict falls in an acute care setting
}

\author{
Peggy Ward-Smith ${ }^{* 1}$, Lynn Barrett ${ }^{2}$, Kristal Rayson ${ }^{3}$, Kourtney Govro ${ }^{3}$ \\ ${ }^{1}$ School of Nursing and Health Studies, the University of Missouri, Kansas, United States \\ ${ }^{2}$ Centerpoint Medical Center, Independence MO, United States \\ ${ }^{3}$ Sphere 3 Consulting, Kansas City, KS, United States
}

Received: August 5, 2014

DOI: $10.5430 /$ cns.v3n1p1
Accepted: October 10, $2014 \quad$ Online Published: October 31, 2014

URL: http://dx.doi.org/10.5430/cns.v3n1p1

\begin{abstract}
Introduction: Aims and objectives: To evaluate the effectiveness that the inclusion of a software program has on the ability to prevent falls among inpatients in an acute care facility. Background: Despite routine assessments and a myriad of interventions, inpatient falls occur at rates between 1.3 and 8.9 per 1,000 patient days. The software used at the study site has the ability to correlate time, patient activity, and unit activity prior to a fall event. Design: A retrospective chart review.
\end{abstract}

Methods: Medical record of 31 randomly selected patients, who experienced a fall while receiving inpatient care at the study site, were reviewed. Demographic, health specific, and activity data were captured. Descriptive and correlational analyses were used to determine if the software program was effective.

Results: The software program, when used in additional to routine assessments, identified a higher-than-normal interruptions for the care provider correlated with a fall. Patients who are prescribed antidepressants constitute a newly identified at-risk population.

Conclusions: Interventions aimed at preventing falls among inpatients need to be multi-faceted, and any assessment needs to be tailored to the specific health and demographic variables of the population. Relevance to clinical practice: Implementation of the software program used at this study site would assist, but not prevent, falls among inpatients, specifically those identified as high-risk.

Key Words: Inpatient falls, Nursing research, Acute care

\section{Introduction}

Inpatient falls has been identified as a concern since the 1950s. ${ }^{[1]}$ In 1957, Thurston ${ }^{[2]}$ noted that, while the incidence of falls among inpatients is not high, the personal consequences and risk of ligation provide incentive to prevent these occurrences. As of October 2008, U.S. hospitals ceased to receive Medicare reimbursement for treatment of fall-related injuries that occurred during an inpatient stay, if the fall was preventable. Despite institution-specific initiatives, the Agency for Healthcare Research and Quality $(\mathrm{AHRQ})^{[3]}$ report that hospital fall rates range from 1.3 to 8.9 per 1,000 patient days. The higher rates occur on inpatient units that provide eldercare, neurology care, and rehabilitative care. Results from a meta-analysis of 17 published randomized controlled trials between 2000 and $2009^{[4]}$ determined that the combined risk ratio for the number of falls as 0.855 . Fall-prevention programs appeared to effectively

\footnotetext{
* Correspondence: Peggy Ward-Smith; Email: wardsmithp@ umkc.edu; Address: School of Nursing and Health Studies, the University of Missouri, Kansas, United States.
} 
reduce falls rates by $14 \%$, albeit with substantial heterogeneity. Appropriate assessment provide a method to identify the patient at risk for falls and the opportunity to implement proactive interventions aimed at decreasing these events.

Aperum ${ }^{\circledR}[5]$ is a computer software system which, when implemented and connected to the bed call light system, in capable of capturing, and correlating, unit activity prior to any fall incidence in an inpatient setting. While the data for this study were collected retrospectively, the Aperum ${ }^{\circledR}$ system is capable of providing real-time data. These data would provide the opportunity to reassign staff and increase the care necessary when a patient exhibits behavior known to correlate to a fall.

Anecdotal data is available which describes the effectiveness of this system in limiting falls among hospital inpatients. ${ }^{[6]}$ However, evaluating the effectiveness of Aperum ${ }^{\circledR}$, and correlating this software program to incidences of falls, using research methods has yet to be done. The purpose of this retrospective chart review study was to determine the ability of this software system to identify situations, or correlate variables, in which an inpatient fall has occurred. These data are a necessary first step in identifying the ability of Aperum ${ }^{\circledR}$ as an intervention proficient in ensuring evidenced based practice is provided.

\section{Background}

The standard definition of a fall is an unexpected event in which the person comes to rest on the ground, floor, or lower level. ${ }^{[7]}$ The definition of an inpatient fall is often uniquely specified by each healthcare facility which varies among institutions. The majority of falls that occur in acute inpatient care settings occur in the patient room area; generally around the bed and/or in bathrooms. The majority of these falls are not observed. ${ }^{[7]}$

The admission of an individual to an institution is a risk factor in and of itself. Age has been cited as a risk factor in the acute care setting, but this is inconsistent. ${ }^{[8]}$ A systematic review of published research by Maike-Lye and associates ${ }^{[9]}$ has identified (1) musculoskeletal disease/weakness, especially hip and/or lower extremity, (2) history of falls, especially multiple falls or injurious fall, (3) gait deficit/use of assistive device, (4) balance deficit, (5) neurological disease, (6) visual deficit, (7) poor nutrition, (8) impaired activities of daily living, (9) cognitive impairment, (10) polypharmacy, (11) hypotension, (12) special toileting needs, and (13) depression as risks for falls, specifically among the inpatient population.

According to the Degelau and colleagues ${ }^{[3]}$ "many reportable falls were related to problems with the fall risk assessment process". Data, obtained in real time, using the Aperum ${ }^{\mathrm{R}}$ system, would allow healthcare providers to identify the patient, determined to be at-risk for a fall, and out of bed without assistance. Research by Shorr and colleagues ${ }^{[10]}$ conclude that the use of bed alarms alone is not sufficient to reduce inpatient falls. According to Lorraine Mion, [11] “... relying on only one intervention to prevent falls, it's very unlikely to be successful" (e1). Bed alarms may be an appropriate intervention, when used with other fall-prevention techniques. Once identified as a person atrisk for falling, a bed alarm may be placed. These devices notify the healthcare providers that the patient is about to have an event. Yet the use of so many alarm devices results in sound dispersion and alarm fatigue among healthcare providers. Thus, the response may be the opposite of what is indicated - the alarm is ignored, resulting in a fall event. The purpose of this study was to determine the ability of an added software program to a bed alarm system on identifying and predicting patients and circumstances that surround falls among inpatients.

\section{Methods}

The study site is a 221 acute care facility located in a suburban area of the Midwest. Survey data indicates that during 2013 this hospital had 13,933 admissions. Prior to initiating any research activity, the Institutional Review Board (IRB) for the health care facility classified the study as minimal risk, no consenting was necessary, and the research protocol was approved. Once this approval was secured, medical record personnel were able to provide inpatient charts for 31 randomly selected individuals who experienced a fall while an inpatient at the study site and were dismissed from the medical center. The Aperum ${ }^{\mathrm{R}}$ software system, which is linked to the call light system, is present on all inpatient beds at the study site, thus randomization to attain the study population was feasible.

Each of these medical records was reviewed; study variable data was entered into a study-specific SPSS file. Study variables included demographic information which was used only to describe the study population. Other study variables included time and location of the fall, admitting circumstances, reason(s) for admission, length of stay, the ambulatory status of the patient, and living arrangements at dismissal. Home medications, as well as medications administered two hours prior to the documented fall were noted and categorized (sedative, analgesic, antidepressant, etc.). If an injury resulted from the fall, the follow-up diagnostic tests and treatment were noted. Correlation of these data to trending information captured by Aperum ${ }^{\circledR}$ was possible using the location and time of the fall. Thus, adherence to the Health Insurance Portability and Accountability Act of 1996 (HIPAA ${ }^{[12]}$ requirements was maintained, and confidentiality of the study data remained intact.

\section{Results}

Demographically, the mean age of patients who experienced a fall while an inpatient at the study site were 60 years (range 
22 to 91 years) and had an average length of stay of 8.4 days (range 1 to 45 days). Discharge status was primarily back to home $(56 \%) ; 25 \%$ of these patients became new admissions to extended care facilities (ECF). Other discharges were back to the ECF, hospice, or against medical advice (AMA). The falls risk assessment, performed as a part of the admission procedures, indicated that $65 \%$ of these patients were identified as a high risk for falls.

All falls occurred within the inpatient room or the private bathroom within each room. Forty percent of the falls occurred while the patient was ambulating (unattended) within their room, $40 \%$ occurred while the patient was performing activities of daily living (unattended) within their private bathroom; the final $20 \%$ of the falls were associated with the patient attempting to get in or out of their hospital bed (unattended). The time of the falls varied, with the greatest incidence of falls $(52 \%)$ occurring either in the early morning (03:30 to $09: 00)$ or the late afternoon (36\%) (13:30 to 19:45).

Analyses of these failed to describe the expected scenario of a fall occurring as a result of a non-response to a call light request. Falls occurred within two hours of a call light request six different times, among four different participants. Two participants had two falls each, after independent call light requests. The majority of this study population $(n=22$; $71 \%$ ) had no order for any type of restraint or environment adaptation (use of a low-bed) as a mechanism to prevent a fall. A low-bed was in use for two of these participants, six had orders for a bed-chair alarm, and one whose admission falls risk assessment identified the need for hourly assessments.

A correlation between administration of ACE inhibitors, anticoagulants, antipsychotics, sedatives and opiates and falls was not apparent. In fact, very few medications were administered two hours prior to the fall. The most frequently medication administered within this time frame was an oral diabetic medication. While the risk of falls is increased when certain medications are prescribed, the British Ministry of Health ${ }^{[13]}$ does not identify oral diabetic medication as a medication with potential risk.

There appears to be trend toward a higher incidence of falls occurring within 48 hours of a planned discharge, but this was not statistically significant $(p=.09)$. Further research, with a larger sample size and among difference acute care inpatient settings are necessary to determine if variations occur among different types of inpatient settings and to determine statistical significance for any of these results.

\section{Discussion}

The use of the nurse call light system, prior to the fall, occurred in only $2 \%$ of these scenarios. Thus, use of the call light, or the ability to respond in a timely manner to a call

Published by Sciedu Press light request, would not have prevented these incidences. Aperum ${ }^{R}$ data determined that, for the four hours prior to the fall, the Nurse Call Light Interruption rate averaged 10.9 per hour for the healthcare provider assigned to the patient who fell. The ability to provide routine hourly rounding is negatively impacted by this rate, causing these researchers to estimate that routine, or hourly rounding would not have prevented greater than $50 \%$ of these falls.

Contrary results related to the use of bed alarms, have been reported by Hubbartt and associates. ${ }^{[14]}$ The use of bed alarms with confused and agitated patients, in the perspective of the nurses, may have helped prevent falls, but even when bed alarms were initiated, hourly monitoring was still needed. When used, alarm fatigue was reported by these nurses, which was described as a consequence of persistently having to respond to an alarm. Over time, these nurses indicated that they simply began to non-respond. Thus, ambivalence, with respect to the use of bed alarms, was a key practice point. Clearly, rigorous research is warranted to identify when the use of a bed alarm system is appropriate for the patient, and helpful to the nurse.

There were no trends or consistencies with the type of medication administered during the two hours prior to the fall. One anecdotal finding was that the majority (62\%) of the study population were receiving antidepressant medication(s) prior to admission. The link between antidepressants and fall risk has received little research attention. Of the published fall assessment tools, only the Hendrich Falls Risk Model II (HFRM-II) ${ }^{[15]}$ includes depression as a variable, which when present, is calculated in to the determination of fall risk.

The inclusion of antidepressant medication to the fall risk assessment appears to have clinical significance in identifying the patient at risk for a fall while an inpatient. This is particularly important for those admitted for inpatient care from an ECF (42\% of the study population), those over age 61 years $(62 \%$ of the study population), or those with a length of stay longer than 8 days ( $46 \%$ of the study population).

\section{Conclusion}

The results of this feasibility study demonstrate the continual need to monitor patients who are receiving care in an acute care setting who are at risk for falls. Implementing the inclusion of antidepressants, as a risk factor for falls, should be considered. While assessing each patient for the presence of known variables which influence falls, additional variables, guided by the specific population, should be added to the assessment. The identification of these specific variables, and including them in the plan of care, provides appropriate, valued, and cost effective care. This is not only evidenced-based-care, but care that will prevent the mortality and morbidity associated with an unintended fall. 


\section{Relevance to clinical practice}

Interventions, aimed at reducing or preventing inpatient falls, should be multifaceted and specific to the patient population. The opinion and perspective of the health care staff that provide care to the patient population needs to be considered in the decision-making process. Adherence to any intervention and responding appropriately depends upon a perceived benefit to the intervention. The ability to identify the patient for whom a bed alarm is appropriate, and will enhance care while decreasing potential adverse effects, is also important. The data obtained in this study begins to identify the patient that would benefit from a bed alarm system, and the impact of a bed alarm system.

\section{References}

[1] Grubel F. Falls: A principal patient incident. Hospital Management. 1959; 88: 37-38. PMid:13829766.

[2] Thurston G. Fatal hospital falls. British Medical Journal (BMJ). 1957; 51: 396-397. http://dx.doi.org/10.1136/bmj.1.501 5.396

[3] Degelau J, Belz M, Bungum L, et al. Prevention of falls (acute care) health care protocol. Bloomington (MN): Institute for Clinical Systems. Improvement (ICSI). 2012; 1-43.

[4] Choi M, Hector M. Effectiveness of intervention programs in preventing falls: a systematic review of recent 10 years and metaanalysis. Journal of the American Medical Directors Association. 2012; 188: e13-21. http://dx.doi.org/10.1016/j.jamda.2 011.04 .022

[5] System iNetwork. Software solutions becomes Aperum. 2003. Available from: http://iprodeveloper.com/development/ software-solutions-becomes-aperum

[6] Healthcare IT News. Sphere3 Aperum demo at HIMSS12. 2012. Available from: http://www.healthcareitnews.com/video /sphere3-aperum-demo-himss12

[7] Lamb SE, Jorstad-Stein EC, Hauer K, et al. Development of a common outcome set for falls injury prevention trials: the Prevention of Falls Network Europe consensus. American Journal of Geriatric Society. 2005; 53: 1618-1622. PMid:16137297. http: //dx.doi.org/10.1111/j.1532-5415.2005.53455.x

[8] Currie LM. Fall and injury prevention. Annual Review of Nursing Research. 2006; 14: 39-74.

[9] Miake-Lye I, Hempel S, Ganz DA, et al. Inpatient fall prevention programs as a patient safety strategy: a systematic review. Annals of Internal Medicine. 2012; 158: 39-396. http://dx.doi.org/10. 7326/0003-4819-158-5-201303051-00005

[10] Shorr RI, Chandler M, Mion LC, et al. Effects of an intervention to increase bed alarm use to prevent falls in hospitalized patients: a cluster randomized trial. Annals of Internal Medicine. 2012; 157: 692-699. http://dx.doi.org/10.7326/0003-4819-157 $-10-201211200-00005$

[11] Mion L. Bed alarms not proven to prevent patient falls in hospitals, UF researchers say. 2012. Available from: http://news .ufl. edu /2012/11/20/bed-alarms/

[12] U.S. Department of Health \& Human Services. Health Information Privacy. 1996. Available from: http://www.hhs.gov/ocr/priv acy/

[13] British Ministry of Health. Medications and the risk of falling. 2011. Available from: http://www.health.gov.bc.ca/preve ntion/pdf/medications-and-the-risk-of-falling.pdf

[14] Hubbartt B, Davis SG, Kautz DD. Nurses' experiences with bed exit alarms may lead to ambivalence about their effectiveness. Rehabilitation Nurse. 2011; 36: 196-199. PMid:21882797. http: //dx.doi.org/10.1002/j.2048-7940.2011.tb00195.x

[15] Swartel KL, Fulton JS, Friesth BM. Relationship between occurrence of falls and fall risk scores in an acute care setting using the Hendrich II fall risk model. MedSurg Nursing. 2013; 22: 180-187. 